\title{
Comparative evaluation of methods for the determination of heat transfer coefficients of liquid and gaseous quenching media
}

\author{
Svetlana Yu. Shevchenko ${ }^{1,2,}$, Yury A. Melnik ${ }^{1}$, Andrey E. Smirnov ${ }^{2}$, and Wai Yan Min Htet ${ }^{2}$ \\ 1 Moscow State University of Technology "STANKIN", 1 Vadkovsky per., 127994 Moscow GSP-4, Russian Federation \\ 2 Bauman Moscow State Technical University, Moscow, Russia
}

Received: 21 September 2017 / Accepted: 20 November 2017

\begin{abstract}
Temperature dependences of heat transfer coefficients of liquid and gaseous quenching media were determined using a gradient probe and prismatic probe of more simple design. The probes of two different designs were tested in the same conditions. Analysis of heat transfer coefficients showed good agreement between the data obtained. The tests were carried out with liquid and gaseous quenching media: water, polymer quenchant, quenching oil and high-pressure nitrogen. Methods of mathematical modeling of steel samples quenching show the adequacy of the results.
\end{abstract}

Keywords: Gas quenching / polymer quenchant / temperature gradient method / enching intensity probe / eat transfer coefficient

\section{Introduction}

Liquid quenching media such as water and oil are featured by the maximum heat transfer coefficient among the whole variety of quenching media. Nevertheless, these media have some disadvantages. In particular, it is difficult to control their cooling ability and, moreover, cooling in a liquid medium is always non-uniform [1-2].

Polymer quenchants provide a possibility to vary the heat transfer coefficient while changing the polymer concentration in the water. Like any liquid, polymer medium is characterized by a non-uniform cooling of the parts being quenched [3-5].

Usage of cooling gaseous high-pressure media enables to easily control their cooling ability by changing the quenching gas pressure. Moreover, such media ensure the maximum cooling uniformity due to the quick and uniform gas flow around the parts being quenched. Such media have an important advantage; they allow not performing the washing of finished parts after the quenching [6-8].

The necessity of experimental determination of quenching medium heat transfer coefficient is caused by the problems of quenching and hardening capacity of parts with certain geometrical dimension as well as by the possibility to apply mathematical modeling to predict quenching results. Experimental evaluation of heat

\footnotetext{
* e-mail: shevchenko@bmstu.ru
}

removal intensity is also important for modeling of other processes, such as the controlled cooling of casts to form the required structure [9].

\section{Materials and methods}

There are methods to determine the heat transfer coefficient [10-11] by measuring the temperature directly at the probe surface and some distance from it. This temperature gradient is proportional to the heat flow from the probe surface during the cooling and the heat transfer coefficient of the surrounding medium; therefore the probes of such design are called gradient probes.

While gradient probe cools, heat flux comes from massive heated probe body and distributes across the thin plate placed on the probe body surface (Fig. 1) [12]. Quenchant takes the heat away from the plate surface. Thermocouple 2 measures temperature at the plate center, thermocouples 1 and 3 measure temperature at its edges. Heat transfer coefficient is calculated according to the formula:

$\alpha=\frac{12 \lambda \delta\left(t_{P}-t_{C}\right)-R^{2} \rho \delta c_{N i}\left(\left(t_{C}^{*}-t_{C}\right)+2\left(t_{P}^{*}-t_{P}\right)\right) / 3 \Delta \tau}{3\left(\frac{t_{P}+2 t_{C}}{3}-t_{0}\right) R^{2}}$.

The probe body (Fig. 1a) and the thermosensitive element (Fig. 1b) are manufactured of X12CrNiTi18-9 steel $(0,12 \% \mathrm{C}, 18 \% \mathrm{Cr}, 10 \% \mathrm{Ni}$, up to $1 \% \mathrm{Ti})$, and the plate at the probe surface is made of nickel. Hot junctions for $\mathrm{K}$-type thermocouples are welded to the plate. 


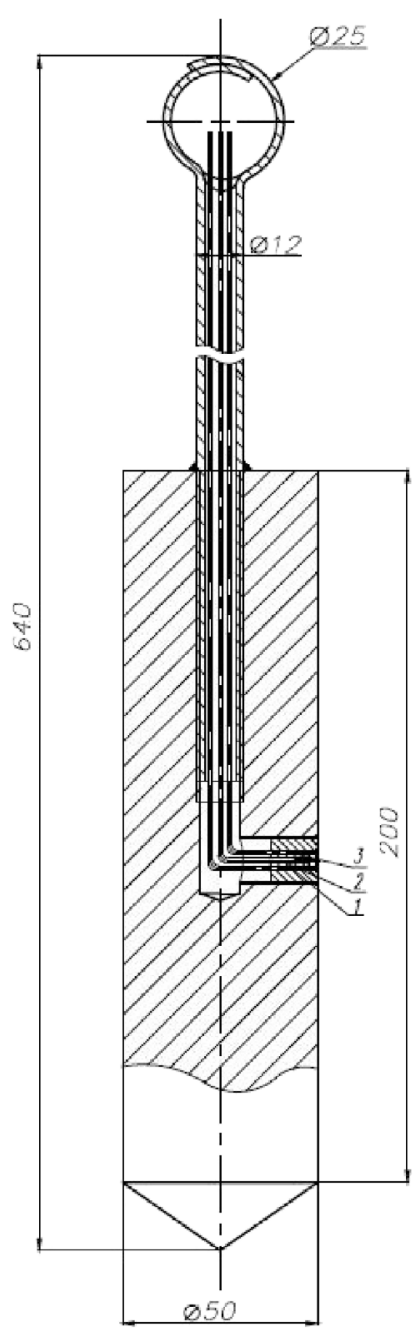

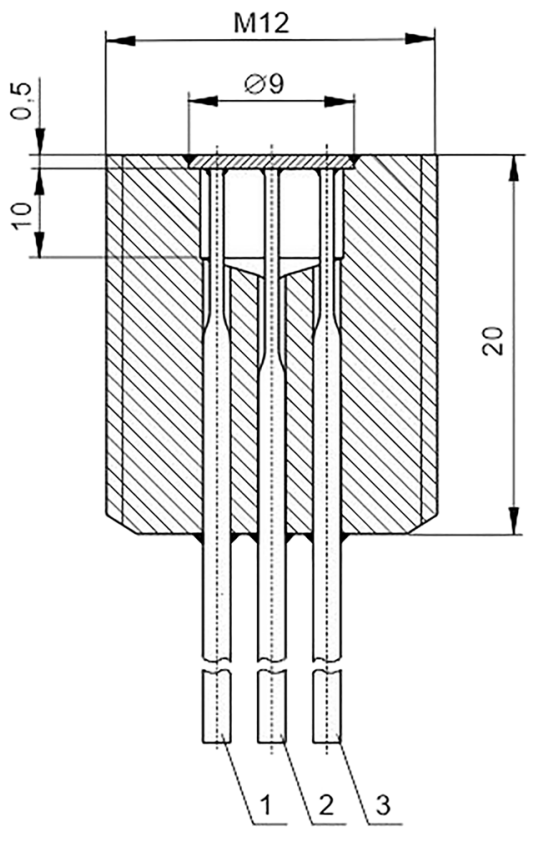

b

Fig. 1. Gradient probe body (a) and thermosensitive element (b)

The paper [13] presents the method to determine the quenchant cooling ability with the help of a prismatic probe. It has been discovered that average surface temperature $\left(t_{\mathrm{s}}\right)$ and average mass temperature $\left(t_{\mathrm{M}}\right)$ isotherms are situated inside while cooling a square prism, a cube, and a short cylinder. Their position in the cross-section of a long square prism is shown in Figure 2a. Coordinates of points $\mathrm{S}$ and $\mathrm{M}$ are situated at the place of intersections of the isotherms and the diagonal of the prism, and the position of these points changes only at the early stage of cooling. At the main stage of the cooling position of points $\mathrm{S}$ and $\mathrm{M}$ stabilizes, and their relative coordinates are $X_{S} / x=0.84$ and $X_{M} / x=0.57$. The advantage of this method is that it is not necessary to place the thermocouple on the surface to measure the probe surface temperature. Heat transfer coefficient $\alpha$ during the quenching cooling is calculated according to the formula:

$$
\alpha=\frac{m c\left(t_{M 1}-t_{M 2}\right)}{F\left(\frac{t_{S 1}+t_{S 2}}{2}-t_{0}\right) \Delta \tau} .
$$

According to [13], the probe was $200 \mathrm{~mm}$ long with the prism side $A=40 \mathrm{~mm}$. Thermocouples were placed along the prism axis and the diagonal distance of $0.84 x$ and $0.57 x$ from it ( $x$ is the length of half the diagonal of the prism) in points S and M. The paper [14] substantiates the reduction of probe size and demonstrates that cooling the side surfaces of the probe does not influence the cooling of lateral faces with the ratio of prism side to its length 1:3. Two probes were manufactured. Dimensions of the first one: prism side $\mathrm{A}=20 \mathrm{~mm}$, prism height $B=60 \mathrm{~mm}$, depth of openings for thermocouples $C=30 \mathrm{~mm}, X_{S}=11.8 \mathrm{~mm}$, $X_{M}=8.1 \mathrm{~mm}$. Dimensions of the second probe: $\mathrm{A}=30 \mathrm{~mm}$, $B=72 \mathrm{~mm}, \quad C=36 \mathrm{~mm}, \quad X_{S}=17.6 \mathrm{~mm}, \quad X_{M}=12.1 \mathrm{~mm}$ (Fig. 2b). The probe bodies were manufactured of X12CrNiTi18-9 steel, and N-type cable thermocouples were placed in the openings with the diameter of $1.5 \mathrm{~mm}$.

Gradient and prismatic probes were tested in liquid quenching media: water, TNK Thermo Oil quenching oil, and the polymer quenchant (15\% solution of KamgidrolZAK [15]). This quenchant consists of polyalkylene oxide, 


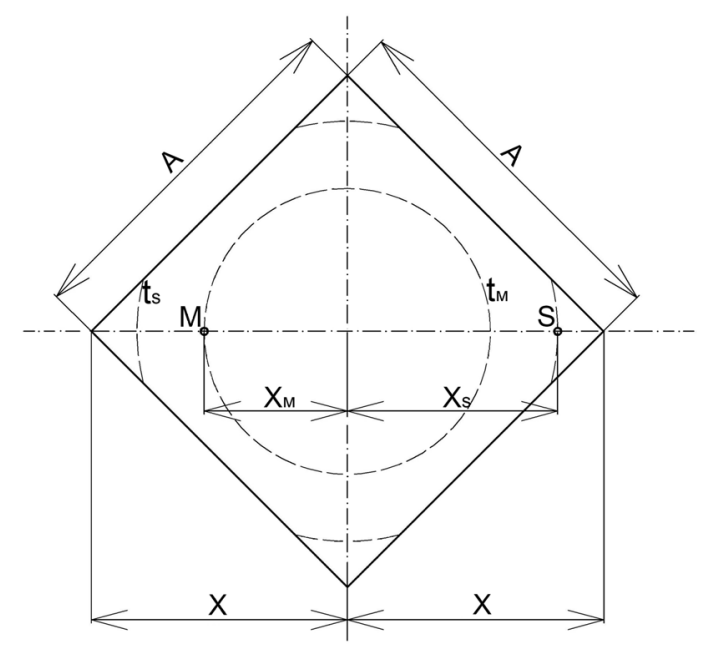

a
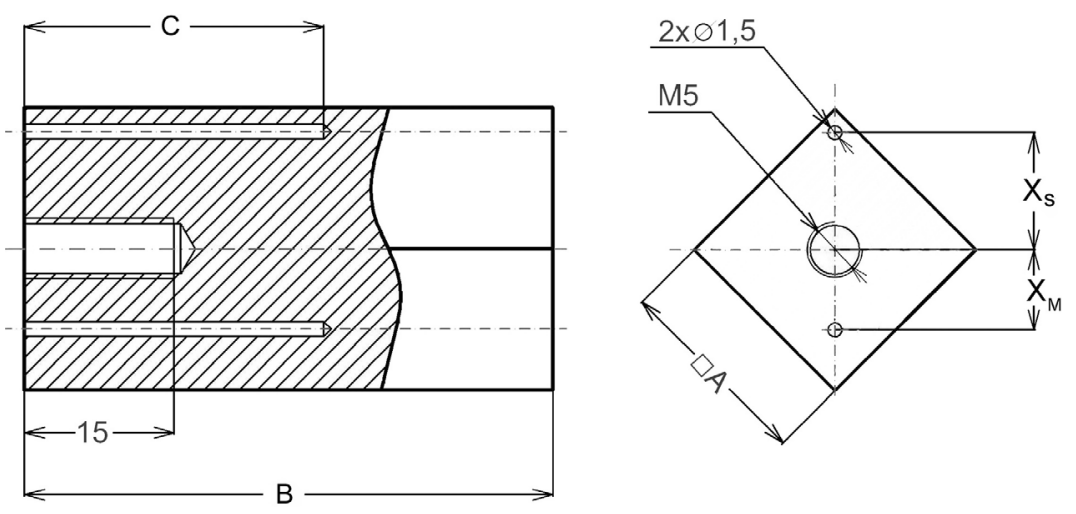

b

Fig. 2. Temperature measuring diagram (a) and prismatic probe body (b)

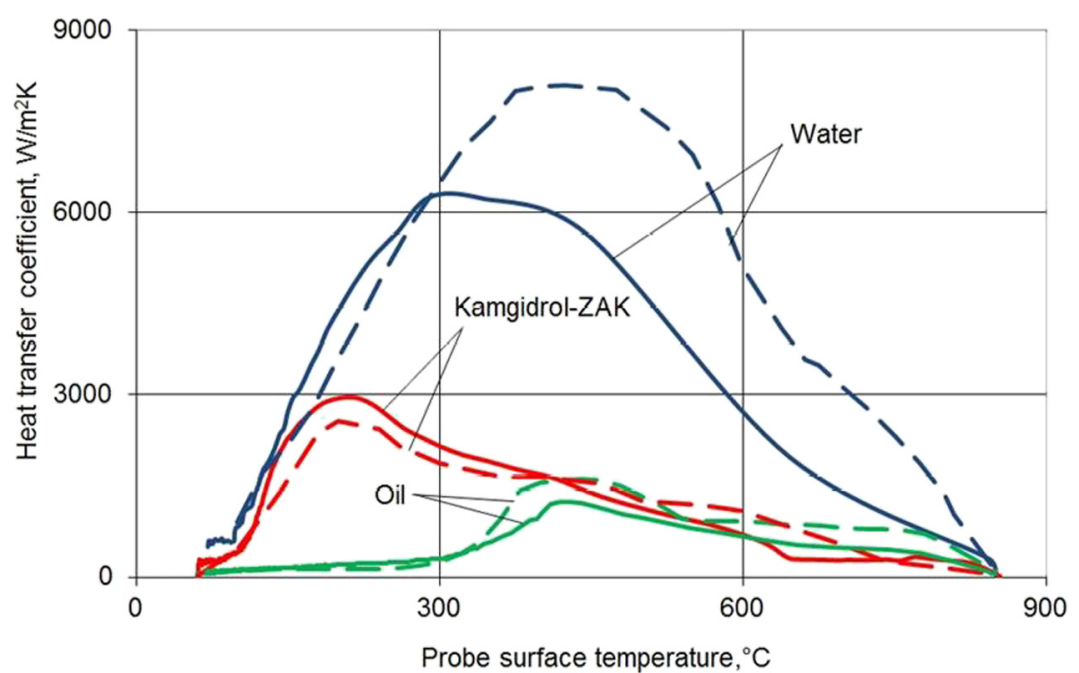

Fig. 3. Heat transfer coefficients of liquid quenchant (solid lines are for gradient probe, dashed lines are for prismatic probe with the side length $30 \mathrm{~mm}$ ) 


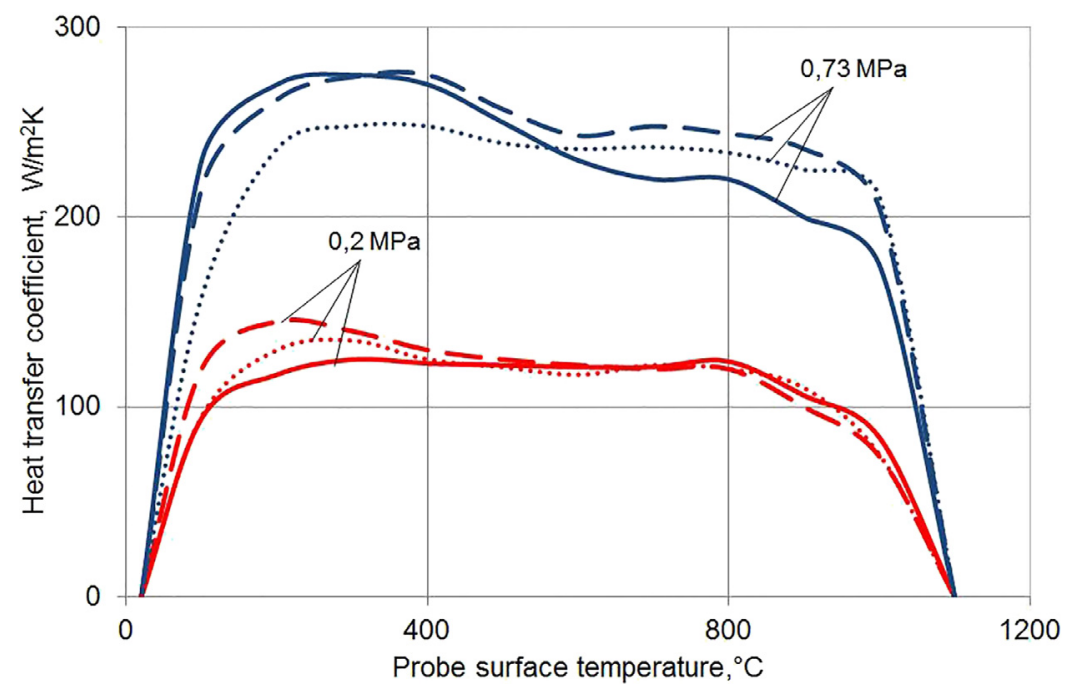

Fig. 4. Heat transfer coefficients of high-pressure nitrogen (solid lines are for gradient probe, dashed lines are for prismatic probe with the side length $30 \mathrm{~mm}$, dotted lines are for prismatic probe with the side length $20 \mathrm{~mm}$ )

water, anticorrosive, antifoaming and bactericide additives. Kamgidrol-ZAK belongs to the group of polymer media with inverse solubility and ensures a more uniform cooling of the parts' surface [16-17]. The probes were heated in the electric pit furnace up to $850^{\circ} \mathrm{C}$, kept until the warm-up across the whole section (gradient was for $100 \mathrm{~min}$, prismatic with the $30 \mathrm{~mm}$ side was for $60 \mathrm{~min}$ ). Then they were cooled in a quenching medium tank, the cooling curves were registered via the computer and an analog-to-digital converter.

To compare the properties of liquid and gaseous quenching media, the cooling ability of high-pressure nitrogen was studied. Gradient and prismatic probes of two-dimension types were heated in the vacuum furnace 10.0VPT-4020/24N manufactured by SECO/WARWICK up to $1100^{\circ} \mathrm{C}$, kept for $100 \mathrm{~min}$ (gradient), $60 \mathrm{~min}$ (prismatic with the side of $30 \mathrm{~mm}$ ) and $40 \mathrm{~min}$ (prismatic with the side of $20 \mathrm{~mm}$ ). Then they were cooled in the nitrogen flow at the pressure of 0.2 to $0.73 \mathrm{MPa}$, with the cooling curves registered.

\section{Results and discussion}

Figure 3 shows temperature dependences of the heat transfer coefficients of the media mentioned above. These coefficients were calculated based on cooling curves of the probes applying formulas (1) and (2). Heat transfer coefficients of water for various sensors differ significantly, because of the instability of cooling properties of this medium. Heat transfer coefficients of oil and KamgidrolZAK differ insignificantly (maximum difference makes $17 \%$ ).

Figure 4 shows temperature dependences of the heat transfer coefficients calculated according to the cooling curves of the three probes. The maximum difference of heat transfer coefficients at the same gas pressure does not exceed $20 \%$.

Analysis of dependences demonstrated in Figures 3 and 4 shows that the maximum heat transfer coefficient of high-pressure nitrogen is several times lower than the heat transfer coefficient of quenching oil.
To estimate the adequacy of the results obtained, the process of quenching cooling of steel cylinders was simulated in ANSYS. The first cylinder was made of $100 \mathrm{Cr} 6$ steel $(1 \% \mathrm{C}, 1.5 \% \mathrm{Cr})$ with the diameter of $20 \mathrm{~mm}$ and the height of $60 \mathrm{~mm}$ was quenched in Kamgidrol-ZAK polymer medium. The second cylinder was made of $20 \mathrm{CrNi} 4$ steel $(0.2 \% \mathrm{C}, 1 \% \mathrm{Cr}, 4 \% \mathrm{Ni})$ with the diameter of $52 \mathrm{~mm}$ and the height of $64 \mathrm{~mm}$ were quenched in nitrogen at the pressure of $0.73 \mathrm{MPa}$. They were produced to perform the comparison with the calculated data. Two thermocouples were placed inside the cylinders in the geometric center and near the lateral surface. Cooling curves were registered during quenching.

Temperature dependences of heat-transfer coefficient of Kamgidrol-ZAK and nitrogen at the pressure of $0.73 \mathrm{MPa}$ were given as boundary conditions for the simulation of steel cylinders of the same size and types of steel quenching. Figure 5 shows the comparison of calculated and experimental cooling curves of 100Cr6 steel cylinder in Kamgidrol-ZAK. Figure 6 shows the comparison of calculated and experimental cooling curves of $20 \mathrm{CrNi} 4$ steel cylinder in nitrogen at the pressure of $0.73 \mathrm{MPa}$. The difference in experimental and calculated curves is insignificant. The reason for the difference is in the thermal effect of martensitic transformation in steels which is difficult to take into consideration for simulation.

Example of practical application of data about the cooling ability of high-pressure nitrogen for predicting the results of quenching of alloyed steel parts is given in [18]. Results obtained during the simulation can be used to predict structural transformations and mechanical properties of parts after quenching [19-20].

\section{Conclusions}

Temperature dependences of heat transfer coefficients for some liquid and gaseous quenching media were obtained using the probes of two different designs. The tests were carried out with liquid and gaseous quenching media. It 


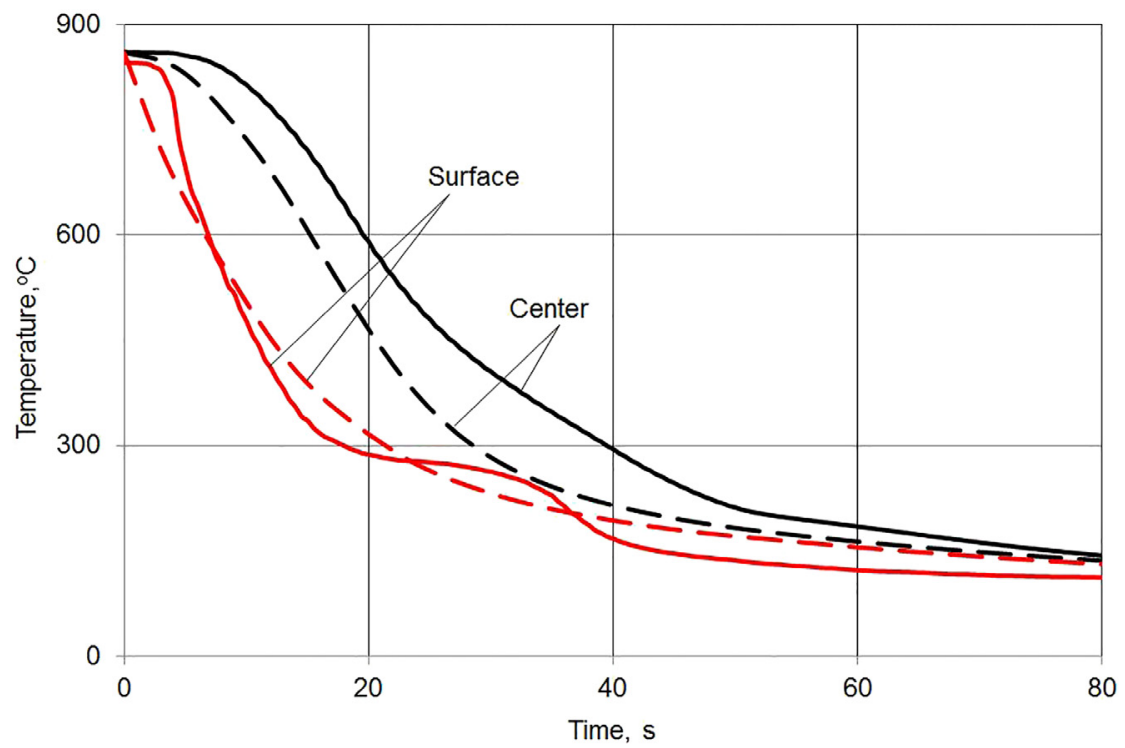

Fig. 5. Calculated (dashed lines) and experimental (solid lines) cooling curves at the center and near the surface of the $100 \mathrm{Cr} 6$ steel cylinder in Kamgidrol-ZAK $15 \%$ solution

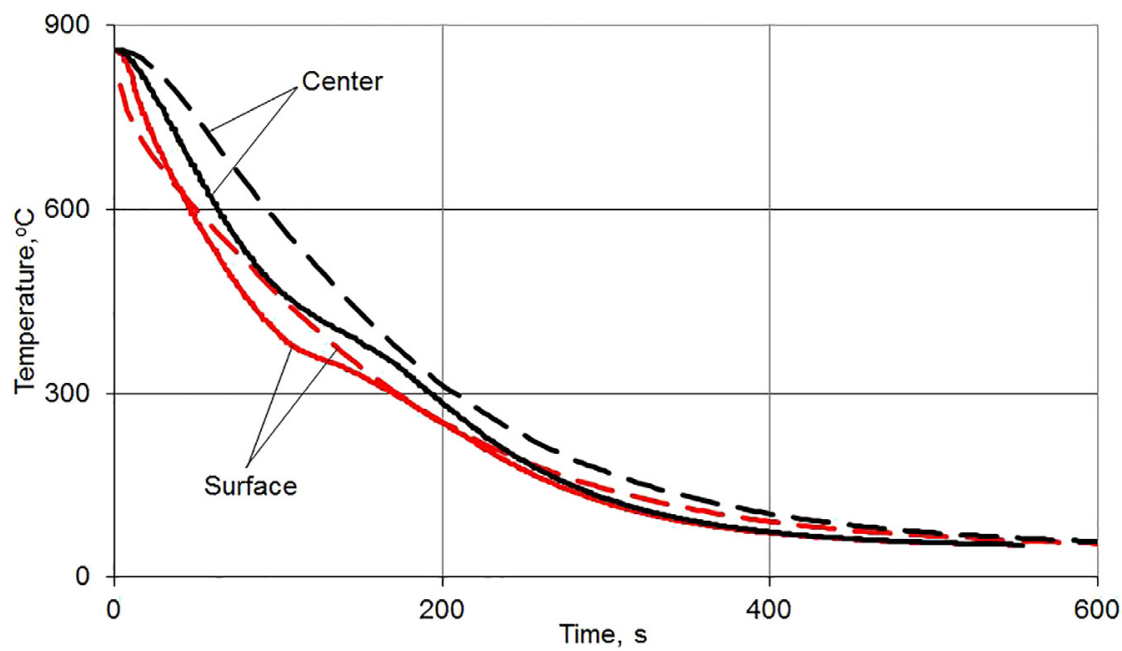

Fig. 6. Calculated (dashed lines) and experimental (solid lines) cooling curves at the center and near the surface of the $20 \mathrm{CrNi} 4$ steel cylinder in nitrogen at the pressure of $0.73 \mathrm{MPa}$

was shown that the maximum heat transfer coefficient of the high-pressure nitrogen at a pressure of $0.73 \mathrm{MPa}$ six times less than the heat transfer coefficient of the quenching oil. It was demonstrated that design and size of the probe do not significantly influence the value of heat transfer coefficient, which is the property of quenching medium itself and not depends on the way of its determination. The maximum difference between two probes results not exceed $20 \%$. Therefore a smaller and simpler probe can be used for experimental determination of heat transfer coefficients.

\section{Nomenclature}

Plate thickness

Calculation range duration, s $\lambda$

\section{$\rho$}

$c_{N}$

$c$

$F \quad$ Probe surface area, $\mathrm{m}^{2}$

$m \quad$ Probe mass, kg

$R \quad$ Plate radius, $\mathrm{mm}$

$t_{\mathrm{M} 1}$ and $t_{M 2}$ Average temperature within probe mass at the beginning and the end of calculation range, ${ }^{\circ} \mathrm{C}$ $t_{S 1}$ and $t_{S 2} \quad$ Average temperature of probe surface at the beginning and the end of calculation range, ${ }^{\circ} \mathrm{C}$ $t_{P}$ and $t_{C} \quad$ Temperature at plate edges and plate center, ${ }^{\circ} \mathrm{C}$ 
$t^{*}$ and $t^{*}{ }_{C} \quad$ Temperature at plate edges and plate center at the previous time step, ${ }^{\circ} \mathrm{C}$

$t_{0} \quad$ Temperature of the environment (quenching medium), ${ }^{\circ} \mathrm{C}$

Acknowledgements. This work was financially supported by the Ministry of Education and Science of the Russian Federation in the framework of the state task in the field of scientific activity of MSTU STANKIN No. 9.7453.2017/6.7.

\section{References}

[1] G.E. Totten, C.E. Bates, M.A. Clinton, Handbook of Quenchants and Quenching Technology, Materials Park, ASM International, 1993

[2] B. Liscic, H.M. Tensi, L.C.F. Canale, G.E. Totten, Quenching Theory and Technology, CRC Press, Taylor \& Francis Group, Boca Raton, Florida, United States 2010

[3] G. Ramesh, K. Narayan Prabhu, Effect of Polymer Concentration on Wetting and Cooling Performance During Immersion Quenching, Metall. end Mater. Trans. B 47 (2016) 859-881

[4] G. Ramesh, K. Narayan Prabhu, Comparative Study of Wetting and Cooling Performance of Polymer-Salt Hybrid Quench Medium With Conventional Quench Media, Exp. Heat Transf. 28 (2015) 464-492

[5] X.F. Chen, L.Y. Zhang, X.H. Jie, Y. Li, X.Y. Huang, Quenching Characteristics Of Glycerol Solution As A Potential New Quenchant, Int. J. Heat Mass Transf. 109 (2017) 209-214

[6] N. Lior, The cooling process in gas quenching, J. Mater. Process. Technol. 155-156 (2004) 1881-1888

[7] Z. Wang, X. Wang, X. Shang, The Effects of Nozzle and Workpiece Placements on Cooling Rate in the Vacuum Highpressure Gas Quenching Furnace Based on CFD, Appl. Mech. Mater. 66-68 (2011) 673-676

[8] V. Heuer, D.R. Faron, D. Bolton, M. Lifshits, K. Loeser, Distortion Control of Transmission Components by Optimized High Pressure Gas Quenching, J. Mater. Eng. Perform. 22 (2013) 1833-1838

[9] A. Yu. Aksenenko, V.N. Klimov, N.V. Korobova, V.I. Luzgin, S. Yu. Shevchenko, Effect of electromagnetic mixing in crystallization on the structure of thixopreforms from castable aluminum alloys, Metal Sci. Heat Treat. 55 (2014) 665-669

[10] B. Liscic, Measurement and Recording of Quenching Intensity in Workshop Conditions Based on Temperature Gradients, Mater. Perform. Charac. 5 (2016) 202-219

[11] B. Liscic, B. Smoljan, Evaluation of the Hardening Capacity of Low-Alloyed Steels Quenched by HPGQ in Vacuum Furnaces, Mater. Sci. Forum 879 (2016) 1807-1812

[12] A.G. Ksenofontov, B.A. Prusakov, S.Y. Shevchenko, Heat flow sensor, RF Patent 2221226. 7G01 K17/20, Byull. Izobr. Polezn. Modeli 1 (2004)

[13] D.V. Budrin, E.L. Sukhanov, V.M. Kondratov, Application of a prism-shape hot probe for determining the cooling capacity of quenching media, Izv. Vysh. Ucheb. Zaved. Chern. Metall. 2 (1966) 134-139

[14] S.Y. Shevchenko, Determination of heat transfer coefficients of quenching media with the help of prismatic sensor, Tekhnol. Met. 5 (2009) 39-42

[15] V.V. Goryushin, S.Y. Shevchenko, On The Use Of Polymer Quenchants In Industry, Metal Sci. Heat Treat. 52 (2010) $255-259$

[16] N.I. Kobasko, Cooling Intensity Of Inverse Solubility Polyalkylene Glykol Polymers And Some Results Of Investigations Focused On Minimizing Distortion Of Metal Components, EUREKA: Phys. Eng. 2 (2017) 55-62

[17] M. Onan, H.I. Ünal, S.H. Atapek, C. Onan, Understanding Of Polymer Quenchant (Polyalkylene Glycol) Characteristic During Quenching Process Of Tool Steels, Int. J. Microstruct. Mater. Prop. 9 (2014) 71-78

[18] S.Y. Shevchenko, A.E. Smirnov, I.V. Kirillov, N.A. Kurpyakova, A Study Of Quenching Cooling In Gaseous Atmospheres, Metal Sci. Heat Treat. 58 (2016) 461-465

[19] J. Jan, E. Prabhu, X. Chen, U. Weiss, Enhanced Heat Transfer Coefficient (HTC) Method to Model Air Quench Process: HTC Patching for More Accurate FEA Temperature Calculation $S A E$, Int. J. Passeng. Cars - Mech. Syst. 9 (2016) 489-496

[20] B. Smoljan, D. Iljkic, G.E. Totten, Mathematical Modeling and Simulation of Hardness of Quenched and Tempered Steel, Metall and Mater. Trans. B 46B (2015) 2666-2673 